\title{
Fatores de risco para o câncer de esôfago: estudo caso-controle em área metropolitana da região Sudeste do Brasil
}

\author{
Risk factors for cancer of the esophagus: a case control study in a \\ metropolitan area of South-Eastern Brazil
}
Suely Godoy Agostinho Gimeno, José Maria Pacheco de Souza, Antonio Pedro Mirra, Pelayo Correa, William Haenszel

\author{
Departamento de Epidemiologia da Faculdade de Saúde Pública da Universidade de \\ São Paulo - Brasil (S.G.A.G., J.M.P. de S.) \\ Registro de Câncer de São Paulo da Faculdade de Saúde Pública da Universidade de \\ São Paulo - Brasil (A.P.M.) \\ Department of Pathology, Louisiana State University Medical Center (P.C.) \\ School of Public Health, Ilinois University (W.H.)
}

\begin{abstract}
A associaçāo do câncer de esôfago com os hábitos de fumar $\theta$ beber, escolaridade e fatores nutricionais foi verificada em um estudo epidemiológico tipo caso-controle, conduzido no Municlplo de Sáo Paulo (Brasil). Oitenta $\theta$ cinco casos foram comparados com 292 controles hospitalares, com diferentes diagnóstlcos, incluslve outros tipos de cåncer. Estimativas brutas, por ponto $\theta$ por intervalo, dos odds ratios, foram obtidas para as variáveis de estudo. Com base nos resultados da análise bruta, foram selecionadas oito variáveis para a utilizaçăo de análise logistica multivariada. O modelo final sugere que tanto o hábito de beber [odds ratio $=3,68$; intervalo com $95 \%$ de confiança $(1,74-7,78)$ ], como o de fumar lodds ratio $=4,86$; intervalo com.95\% de conflança $(1,95-12,13)]$ e o consumo freqüente de pimenta [odds ratio $=2,48$; intervalo com $95 \%$ de confiança $(1,46 \cdot 4,23)$ ] săo fatores de risco importantes para a doença. Obteve-se odds ratio $3,43(1,31 \cdot 8,97)$ para o hábito de fumar cigarro comum e odds ratio $4,18(1,38 \cdot 12,66)$ para cigarro de palha.
\end{abstract}

Neoplasias esofágicas, epidemiologia. Fatores de risco.

O câncer de esôfago pode ser visto como um dos mais importantes sob o ponto de vista médico, devido à sua alta fatalidade e à frequiencia com que ocorre em todo o mundo. É um câncer comum no norte da China, no cinturảo que se estende da Rússia européia ao nordeste do Mar Cáspio, Turquia, Irãa, até o centro da Ásia. É também comum na população negra do Sudeste da África. Taxas intermediárias são encontradas entre negros americanos, em regiōes da França, Suiça, Japão e
América Latina, destacando-se o Uruguai, Argentina e Brasil ${ }^{31}$. Estudos realizados com migrantes japoneses em diferentes áreas geográficas revelam que a variação nas taxas de incidência de câncer de esôfago é maior para homens ${ }^{28}$.

Esse câncer é raro na juventude e tem rápido aumento da incidência em idades mais avançadas, sugerindo como causa a exposição prolongada a agente(s) carcinogênico(s) do ambiente externo ${ }^{8}$. Há grande variabilidade na razão de taxas de

Separatas/Reprints: José Maria Pacheco de Souza - Departamento de Epidemiologia da Faculdode de Saúde Pública da Universidade de Säo Paulo - Av. Dr. Amaldo, 715 - 01246-904 - Sdo Paulo, SP - Brasil - Fax: (011) 282.2920

Ediçäo subvencionado pela FAPESP. Processo 95/2290-6.

Recebido em 19.9.1994. Aprovado em 10.3.1995. 
incidência entre os sexos. Em 1971, Cook ${ }^{6}$ obteve as razōes ajustadas de incidências 1:1 a 13:1 para câncer de esôfago, em homens e mulheres com idade entre 35 e 64 anos que residiam em diversas regiōes da África. Em estudo caso-controle, realizado em São Paulo no ano de 1991, sobre etnia e risco de câncer, Bouchardy e col. ${ }^{3}$ mostraram que negros e mulatos têm maior chance de desenvolver câncer de esôfago que os brancos, sendo o odds ratio ajustado igual a $2,6(2,2-3,2)$ e $1,5(1,2$ $1,8)$, respectivamente.

A maioria dos tumores de esôfago é do tipo epidermóide. Os adenocarcinomas, mais raros, originam-se de focos ectópicos da mucosa gástrica. O tumor se desenvolve sob a forma de crescimento exofítico ou lesão ulcerada. Em geral, se estende superficialmente sob a mucosa e submucosa; a difusăo é rápida e às vezes atinge profundamente a parede da víscera, o que é facilitado pela falta de cobertura serosa. É frequiente a invasăo de importantes estruturas vizinhas ${ }^{22,31}$.

$\mathrm{Na}$ maioria dos casos a disfagia leva ao diagnóstico mas, infelizmente, é um sintoma tardio que não surge até que haja obstruçåo evidente; o prognóstico é mau. Apesar de cirurgias agressivas e radioterapia, as taxas de sobrevida de cinco anos permanecem inferiores a 5\%. Essas taxas sāo de $15 \%$ para tumores operáveis no terço superior do esôfago, $6 \%$ para o terço médio e $1 \%$ para o terço inferior ${ }^{22,31}$.

$\mathrm{O}$ aspecto etiológico dessa doença é de grande complexidade; parece que é causado por vários agentes, em diferentes localidades. A incidencia depende do potencial cancerígeno de fatores individuais ou da combinação deles, além da quantidade de contaminantes ambientais a que uma população em particular esteja exposta. O papel de diferentes fatores tem sido estudado: a influência de fatores genéticos, condiçōes anormais do esôfago, hábitos tais como beber, fumar e mascar, consumo de alimentos em temperaturas extremas, dietas, condição socioeconômica e fatores ocupacionais, além do efeito de radiações ionizantes, da composição do solo, do clima e da vegetação. As evidências apontam para uma doença que é característica de locais com precárias condiçōes socioeconômicas e dieta deficiente e que tanto o álcool como o fumo têm papel importante no aparecimento do tumor em

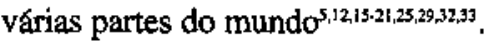

No período de 1979 a 1981 foi conduzido um estudo caso-controle no Município de São Paulo com o objetivo de verificar a associação do câncer de esôfago com possíveis fatores de risco, como parte de um projeto que também incluia o estudo do câncer de reto. Na presente pesquisa, os dados do estudo sobre câncer de esôfago foram trabalhados apenas parcialmente e julga-se oportuno, agora, reexaminar essa informaçōes com a utilizaçāo de técnicas estatisticas mais poderosas, tais como a análise multivariada com regressão logística.

\section{Material e Método}

As informaçőes foram coletadas em dez hospitais do Município de São Paulo, que atendiam pacientes com câncer e concordaram em participar do estudo.

\section{Caracterlzação da População Estudada}

Foi considerado caso todo indivíduo com diagnóstico de câncer de esôfago comprovado histologicamente, que se encontrasse internado ou freqüentando $o$ ambulatório dos hospitais onde o estudo foi desenvolvido.

Todo doente, quer fosse de ambulatorio, quer estivesse internado, preferivelmente no mesmo hospital onde ocorreu o caso, que não apresentasse câncer de laringe, boca, bexiga ou pulmão, pareado com o caso, segundo sexo e idade ( \pm 5 anos), foi considerado um controle.

Para o estudo atual, além dos cem controles pareados com os primeiros cem casos de esôfago, foram inclúfos, no grupo de controle, os casos de reto e seus respectivos controles, sendo excluídos 4 pares em funçāo de apresentarem idade superior a 80 anos (Tabela 1).

Dos cem indivíduos inicialmente incluídos no grupo de casos com câncer de esôfago, quinze não tiveram seu diagnóstico confirmado pelo exame histologico e foram excluídos do estudo; dessa forma, a amostra final ficou composta por 85 casos com câncer de esôfago e 292 controles.

\section{Caracterização do Método de Estudo}

Foi utilizado questionário composto por elementos de identificaçăo e questões referentes aos fatores de risco estudados; as informaçסes sobre os aspectos clínicos foram obtidas mediante consulta ao prontuário médico. As entrevistas dos pacientes foram feitas por médicos treinados e supervisionados pela equipe que coordenou a pesquisa.

A existência de associaçāo entre o câncer de esôfago e cada um dos fatores foi verificada, con- 
Tabela 1 - Número e percentagem de controles, segundo diagnostico hospitalar. Săo Paulo, 1994.

\begin{tabular}{lrr} 
Diagnóstico hospitalar & $n^{2}$ & $\%$ \\
\hline Neoplasias em geral (câncer de reto e outros tipos de cåncer*) & 195 & 66,8 \\
Doenças parasitárias e infecciosas & 5 & 1,7 \\
Doenças de glándulas endócrinas, da nutrição e do metabolismo & 4 & 1,4 \\
Doenças do sistema nervoso e dos órgãos do sentido & 4 & 1,4 \\
Doenças do aparelho circulatório & 12 & 4,0 \\
Doenças do aparelho respiratório & 2 & 0,7 \\
Doenças do aparehtho digestivo & 39 & 13,4 \\
Doenças do aparelho gênito-urinário & 8 & 2,7 \\
Doenças da pele & 1 & 0,3 \\
Doenças do sistema osteomuscular e do tecido conjuntivo & 16 & 5,5 \\
Lesőes e envenenamento & 2 & 0,7 \\
Não especificado & 4 & 1,4 \\
\hline Total & 292 & 100
\end{tabular}

- Năo foram selecionados como controles pacientes com câncer de boca, bexiga, laringe e pulmão

siderando as variáveis em sua forma dicotômica (Tabela 2). Foram obtidas estimativas dos odds ratios por ponto e por intervalo, além do valor da estatística qui-quadrado de Mantel e Haenszel $\mathrm{X}^{2} \mathrm{mh}$; examinou-se também a existência de relação dose-resposta entre o hábito de beber e de fumar e o risco de desenvolver câncer de esôfago, segundo três níveis de exposiçāo ao fumo e à bebi$\mathrm{da}^{4}$. Foram selecionadas as variáveis cujo nível descritivo de significância do teste $(\mathrm{P})$ foi menor do que $0,20^{14}$. Foram considerados como efeitos de principal interesse tanto o hábito de fumar como o de beber. A técnica de regressão logística não condicional foi utilizada nas etapas seguintes da análise com o objetivo de controlar a possível ação de confusão ou modificação de efeito exercida por outras variáveis sobre as associaçðes de interesse. $O$ processo de modelagem estatística foi iniciado pelo modelo saturado, com eliminação posterior daquelas variáveis com $P$ maior ou igual a $5 \%$.

Tabela 2 - Forma dicotómica das variáveis consideradas na análise. Săo Paulo, 1984.

\begin{tabular}{|c|c|c|}
\hline \multirow[b]{2}{*}{ Variável } & \multicolumn{2}{|c|}{ Condiçăo } \\
\hline & $\begin{array}{l}\text { Não exposto } \\
\text { (nivel basal) }\end{array}$ & Exposto \\
\hline $\begin{array}{l}\text { Sexo } \\
\text { Idade } \\
\text { Local de residencia } \\
\text { Religiăo } \\
\text { Antecedentes familiares de cáncer } \\
\text { Escolaridade } \\
\text { Local de nascimento } \\
\text { Zona de nascimento } \\
\text { Consumo freqüente de carne salgada } \\
\text { Consumo freqūente de carne embutida } \\
\text { Auséncia de consumo de verduras } \\
\text { Auséncia de consumo de frutas } \\
\text { Consumo freqüente de pimenta } \\
\text { Hábito de fumar } \\
\text { Hábito de beber (destilados) }\end{array}$ & $\begin{array}{l}\text { Feminino } \\
20 \text { a } 57 \text { anos } \\
\text { Mun. de São Paulo } \\
\text { Católlca } \\
\text { Não } \\
\text { Demals } \\
\text { Estado de São Paulo } \\
\text { Urbana } \\
\text { Náo come } \\
\text { Năo come } \\
\text { Diário } \\
\text { Díárlo } \\
\text { Não } \\
\text { Nunca fumou } \\
\text { Nunca bebeu }\end{array}$ & $\begin{array}{l}\text { Masculino } \\
58 \text { a } 60 \text { anos } \\
\text { Outros locais } \\
\text { Outras } \\
\text { Sim } \\
\text { Analfabeto } \\
\text { Outros locals } \\
\text { Rural } \\
\geq 1 \text { vez por mes } \\
\geq 1 \text { vez por mess } \\
\leq 6 \text { vezes por semana } \\
\leq 6 \text { vezes por semana } \\
\geq 1 \text { vez por mês } \\
\text { Fuma (atual ou passado) } \\
\text { Bebe (atual ou passado) }\end{array}$ \\
\hline
\end{tabular}

Todos os fatores considerados na análise com o modelo de regressão logística estavam sob forma dicotômica (Tabela 2).

No modelo final obtido, substituiu-se a variável hábito de furmar pelos fatores hábito de fumar cigarro comum (independentemente de filtro) e hábito de fumar cigarro de palha, visando conhecer o comportamento do risco em relação a esses dois vícios. Foram eliminados para essa análise todos os indivíduos que tivessem o hábito 
de fumar outros tipos de fumo que não aqueles de interesse (fumantes de cachimbo: 19 casos e 21 controles; fumantes de charuto: 4 casos e 4 controles), ficando, dessa forma, a amostra composta por 329 indivíduos ( 62 casos e 267 controles).

A qualidade do ajuste do modelo foi verificada pelo exame dos valores da estatística deviance. Foram utilizados os programas Epi Info' Egret e Multr"1".

\section{Resultados}

$\mathrm{Na}$ Figura são apresentados os casos com câncer de esôfago segundo localização anatômica do tumor. Observou-se predominância do tumor nos terços médio e inferior do esôfago, sendo estes principalmente do tipo carcinoma de células escamosas. Na Tabela 3 encontra-se a distribuição de casos e controles segundo sexo e idade. Observa-se que a doença foi mais frequiente no sexo masculino (4,3 homens para cada mulher).
Nas Tabela 4 estão apresentados os resultados da análise bruta (forma dicotômica).

Apresentaram significância estatística $(\mathrm{P}<0,20)$ as variáveis sexo, local de nascimento, consumo frequiente de came salgada, consumo frequiente de came embutida, ausência de consumo de frutas, consumo frequiente de pimenta, o hábito de beber destilados e o de fumar.

Os resultados da análise de gradação dos odds ratios para o hábito de beber e o de fumar segundo três níveis de exposição estão apresentados na Tabela 5. Destacam-se os valores dos odds ratios para os níveis de maior exposição a esses fatores.

Nas Tabelas 6 e 7 são apresentados os modelos finais obtidos a partir da utilizaçảo da análise multivariada. A análise bruta sugeriu risco aumentado da doença entre indivíduos com consumo habitual de carne salgada e embutida, com ausência de consumo de frutas e que nasceram fora do Estado de Såo Paulo, porém esses riscos nāo mantiveram a significância estatística quando se fez o controle simultâneo para o hábito de beber, de fumar e o consumo de pimenta.

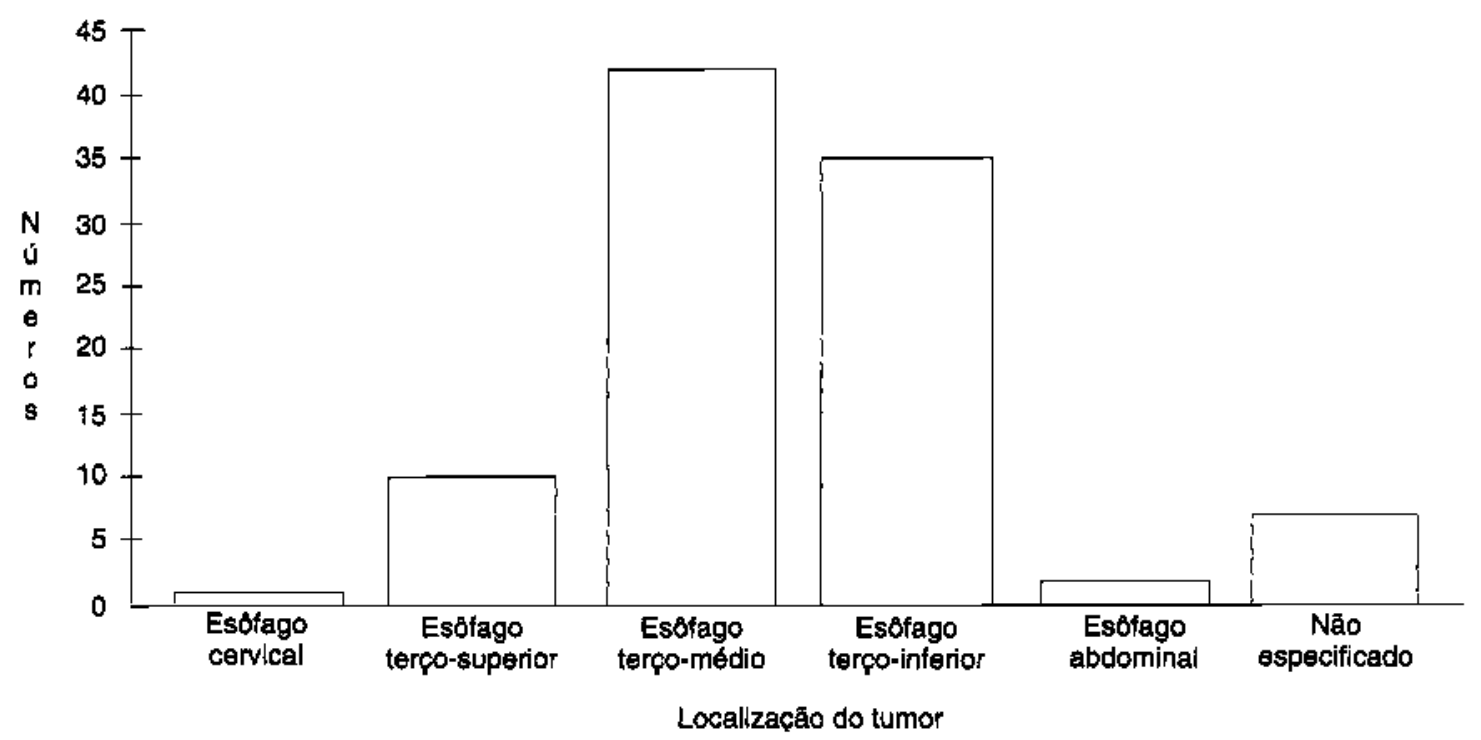

Figura - Número de casos com câncer de esófago, segundo localizaçāo anatômica do tumor. Sāo Paulo, 198 \{

Tabela 3 - Número e percentagem de casos e controles, segundo sexo e idade (anos). São Paulo 1981.

\begin{tabular}{|c|c|c|c|c|c|c|c|c|c|c|}
\hline \multirow{3}{*}{ Idade } & \multicolumn{4}{|c|}{ Casos } & & & \multicolumn{4}{|c|}{ Controles } \\
\hline & \multicolumn{2}{|c|}{ Masculino } & \multicolumn{2}{|c|}{ Feminino } & \multicolumn{2}{|c|}{ Masculino } & \multicolumn{2}{|c|}{ Feminino } & \multicolumn{2}{|c|}{ Total } \\
\hline & $\mathrm{N}^{2}$ & $\%$ & $\mathrm{~N}^{2}$ & $\%$ & $N^{0}$ & $\%$ & $\mathrm{~N}^{\mathrm{e}}$ & $\%$ & $N^{E}$ & $\%$ \\
\hline $\begin{array}{l}20 \text { a } 57 \\
58 \text { a } 80\end{array}$ & $\begin{array}{l}43 \\
26\end{array}$ & $\begin{array}{l}62,3 \\
37,7\end{array}$ & $\begin{array}{l}8 \\
8\end{array}$ & $\begin{array}{l}50,0 \\
50,0\end{array}$ & $\begin{array}{l}87 \\
76\end{array}$ & $\begin{array}{l}53,4 \\
46,6\end{array}$ & $\begin{array}{l}72 \\
57\end{array}$ & $\begin{array}{l}55,8 \\
44,2\end{array}$ & $\begin{array}{l}210 \\
167\end{array}$ & $\begin{array}{l}55,7 \\
44,3\end{array}$ \\
\hline Total & 69 & 100 & 16 & 100 & 163 & 100 & 129 & 100 & 377 & 100 \\
\hline
\end{tabular}


Tabela 4 - Resultados da análise bruta (variáveis sob a forma dicotômica).

\begin{tabular}{|c|c|c|c|c|}
\hline Variável & Odds ratio & $\begin{array}{l}\text { Intervalo com } 95 \% \text { de } \\
\text { confiança }\end{array}$ & $x^{2}$ & $P$ \\
\hline $\begin{array}{l}\text { Sexo } \\
\text { idade } \\
\text { Local de residéncia } \\
\text { Religiäo } \\
\text { Antecedentes familiares de câncer } \\
\text { Escolaridade } \\
\text { Local de nascimento } \\
\text { Zona de nascimento } \\
\text { Consumo freqüente de came salgada } \\
\text { Consumo freqüente de came embutida } \\
\text { Ausência de consumo de verduras } \\
\text { Ausencia de consumo de frutas } \\
\text { Consumo freqüente de pimenta } \\
\text { Hábito de fumar } \\
\text { Hábito de beber (destilados) }\end{array}$ & $\begin{array}{l}3,41 \\
0,80 \\
1,14 \\
0,89 \\
0,65 \\
0,68 \\
0,70 \\
1,17 \\
2,05 \\
1,51 \\
1,15 \\
1,81 \\
2,98 \\
8,93 \\
9,19\end{array}$ & $\begin{array}{l}1,82-6,49 \\
0,47-1,35 \\
0,67-1,91 \\
0,39-1,98 \\
0,31-1,36 \\
0,15-2,59 \\
0,42-1,18 \\
0,70-1,97 \\
1,11-3,77 \\
0,84-2,71 \\
0,69-1,94 \\
1,07-3,07 \\
1,75-5,08 \\
3,58-23,73 \\
4,12-21,00\end{array}$ & $\begin{array}{l}17,83 \\
0,82 \\
0,26 \\
0,09 \\
0,14 \\
0,02 \\
2,07 \\
0,42 \\
6,33 \\
2,26 \\
0,34 \\
5,60 \\
19,33 \\
33,00 \\
42,11\end{array}$ & $\begin{array}{l}0,00 \\
0,37 \\
0,61 \\
0,76 \\
0,71 \\
0,89 \\
0,15 \\
0,51 \\
0,01 \\
0,13 \\
0,56 \\
0,02 \\
0,00 \\
0,00 \\
0,00\end{array}$ \\
\hline
\end{tabular}

Em itálico estāo apresentadas as varlávels que foram selecionadas para o desenvolvimento da análise multivariada

Referências sobre a possível existência de interação entre o álcool e o fumo são encontradas na literatura; no presente trabalho o termo de interaçāo entre estes dois fatores nåo foi mantido no modelo final por provocar distorçōes nos resultados (sugetindo possível açāo protetora do álcool e do fumo nos estratos de nâo fumantes e năo bebedores, respectivamente), em virtude do pequeno número de casos expostos a bebida no estrato de não fumantes.

\section{Comentários}

Os resultados da análise multivariada sugerem que o álcool, o fumo e o consumo frequiente de pimenta são fatores de risco independentes para a doença. A evidente relação dose-resposta para os diferentes níveis de exposição ao fumo e à bebida fortalece a hipótese de que esses fatores têm importante papel na etiologia dessa doença. Achados semelhantes foram encontrados por outros pesquisadores ${ }^{1,16,23,24,27,32,34}$. Tuyns ${ }^{30}$ (1990) destaca que o álcool é um dos poucos exemplos de substâncias químicas que parecem estar claramente envolvidas em certos tipos de câncer em humanos, especialmente os de boca, laringe, hipofaringe $e$ esôfago, sem ter sido possível demonstrar esse fato em estudos experimentais.

$B 1 t^{2}$ (1992) sugere algumas hipotese sobre os possíveis mecanismo de açâo do álcool na produçảo do câncer; o álcool poderia:

1) ser veículo para algumas substâncias reconhecidamente cancerígenas (aditivos, por exemplo);

2) gerar produtos metabólicos que são carcinogênicos para o homem;
Tabela 5 - Resultados da análise de gradação do odds ratios.

\begin{tabular}{|c|c|c|c|}
\hline Variável & $X^{2}$ & $\mathbf{P}$ & odds ratio \\
\hline $\begin{array}{l}\text { Quantidade de cigarro } \\
\text { Nāo fuma (nivel 0) } \\
\leq 20 \text { clgarros por dia (nivel 1) } \\
>20 \text { cigarros por dia (nivel 2) }\end{array}$ & 20,08 & $\begin{array}{l}0,00 \\
1,51 \\
5,57\end{array}$ & $1, \infty$ \\
\hline $\begin{array}{l}\text { Quantidade de bebida } \\
\text { Não bebe (nivel } 0 \text { ) } \\
\leq 4 \text { doses por dia (nivel 1) } \\
>4 \text { doses por dia (nivel 2) }\end{array}$ & 61,65 & 0,00 & $\begin{array}{r}1,00 \\
7,05 \\
15,03\end{array}$ \\
\hline
\end{tabular}

3) atuar como solvente, facilitando a penetração de substâncias cancerígenas (como as do tabaco, por exemplo) nos tecidos, especialmente da boca e esôfago;

4) reduzir a ingeståo e a biodisponibilidade de certos nutrientes protetores;

5) aumentar a exposição celular a agentes oxidantes, aumentando o risco de desenvolvimento de câncer;

6) diminuir a resposta imune, dado seu efeito sobre o estado nutricional.

Esteve e col. ${ }^{10}(1984)$ afirmam que agentes cancerígenos, tanto indutores como promotores, sāo

Tabela 6 - Resultado final da análise com o modelo de regressão logística nāo condicional.

\begin{tabular}{lcl}
\hline Variável & $\hat{\beta} \begin{array}{l}\text { Odds } \\
\text { ratio }\end{array}$ & $\begin{array}{l}\text { Intervalo com } \\
95 \% \text { de contlança }\end{array}$ \\
\hline $\begin{array}{l}\text { Hábito de beber } \\
\text { Hábito de fumar } \\
\text { Consumo freqüente }\end{array}$ & $1,303,68$ & $1,74-7,82$ \\
de pimenta & $0,91,86$ & $1,95-12,13$ \\
\hline
\end{tabular}


Tabela 7 - Resultado final da análise com o modelo de regressão logística nāo condicional (cigarro comum $\theta$ çigarro de palha).

\begin{tabular}{|c|c|c|c|}
\hline Variável & $\hat{\beta}$ & Odds ratio & $\begin{array}{l}\text { Intervalo com } 95 \% \text { de } \\
\text { confiança }\end{array}$ \\
\hline $\begin{array}{l}\text { Hábito de beber } \\
\text { Hábito de fumar cigarro de comum (excluslvo)" } \\
\text { Hábito de fumar cigarro palha (exclusivo)* } \\
\text { Hábito de fumar cigarro comum e cigarro de }\end{array}$ & $\begin{array}{l}1,48 \\
1,23 \\
1,43\end{array}$ & $\begin{array}{l}4,40 \\
3,43 \\
4,18\end{array}$ & $\begin{array}{r}1,86-10,40 \\
1,31-8,97 \\
1,38-12,66\end{array}$ \\
\hline $\begin{array}{l}\text { palha ((simultâneo) } \\
\text { Comsumo freqüente de pimenta }\end{array}$ & $\begin{array}{l}2,63 \\
0,74\end{array}$ & $\begin{array}{r}13,96 \\
2,09\end{array}$ & $\begin{array}{l}3,31 \cdot 58,96 \\
1,13 \cdot 3,88\end{array}$ \\
\hline
\end{tabular}

* Em relaçăo a năo fumantes

encontrados no tabaco e essa presença é suficiente para explicar a induçāo do câncer no homem.

A importância dos diferentes tipos de fumo tem sido investigada por diferentes pesquisadores ${ }^{10.13 .26}$. Esteve e col. ${ }^{10}(1984)$ apontam que um dificuldade para o estudo do risco de acordo com o tipo de fumo é a pequena prevalência de fumantes de alguns tipos de cigarro. No presente trabalho encontrou-se maior odds ratio para o hábito de fumar cigarro de palha em relação a não fumantes; destaca-se que a amplitude do intervalo de confiança obtido para essa variável sugere instabilidade da medida de efeito encontrada lodds ratio: 13,96; intervalo com $95 \%$ de confiança: $(3,31-58,96)]$. O odds ratio para a exposição conjunta do hábito de fumar cígarro comum e de fumar cigarro de palha em relação a não fumantes não sugere interação entre esses dois hábitos.

A pimenta é um agente que pode levar à irritação da mucosa do trato gastrointestinal. $O$ fato do consumo habitual deste alimento ter se revelado como um fator de risco importante para a doença pode talvez ser explicado pela possível ação agressiva desse alimento sobre a mucosa do esôfago.

\section{Agradecimento}

A um dos relatores que apreciaram o manuscrito, pelas valiosas observaçס̋es e sugestðes.

\section{Refere̊ncias Blbliograficas}

1. BARRA, S.; FRANCESCHI, S.; NEGRI, E.; TALAMINI, R.; VECCHIA, C. Type of alcoholic beverage and cancer of the oral cavity, pharynx and oesophagus in an Italian area with high wine consumption. Int. J. Cancer, 46: 1017$20,1990$.

2. BLOT, W.J. Alcohol and cancer. Cancer Res., 52 : $2119 \mathrm{~s}-2123 \mathrm{~s}, 1992$.

3. BOUCHARDY, C.; MIRRA, A.P.; KHLAT, M.; PARKIN, M.D; SOLZA, J.M.P.; GOTLIEB, S.L.D. Ethnicity and cancer risk in Sâo Paulo - Brazil. Cancer Epidemiol. Biom. \& Prev. 1:22-7, 1991.

4. BRESLOW, N.E, \& DAY, N.E. Statiscals metbods in cancer researcb: the analysis of case-control studies. Lion, IARC, 1980.v.1 (Scientific Publication, n. 12).

5. CHENG, K.K.; DAY, N.E.; DUFFY , S.W.; LAMT, H.; FOX, M.; WONG, J. Pickled vegetables in the aetiology of oeosopfhageal cancer in HongKong Chinese. Lancet, 339:1314-8, 1992.

6. COOK, P.J. Cancer of esophagus in Africa, Br.J. Cancer, 25:853-80, 1971

7. DEAN, J.; DEAN, A.; BURTON, A.; DICKER, R. Ept Info computer programs for epidemiology.
Atlanta, Division of Surveilance and Epidemiologic Studies, Epidemiology Program Office, Center of Disease Control, 1990.

8. DOLL, R. \& PETO, R. The causes of cancer quantitative estimatives of avoidable risk of cancer in United States today. J. Natl. Cancer Inst., 66:1193-308, 1981

9. EGRET: Epidemiologial graphics, estimation and testing package. Seatle, Washington, D.C., Statistics and Epidemiology Research Corporation, 1988.

10. ESTEVE, J.; TUYNS, A.J.; RAYMOND, L.; VINEIS, P. Tobacco and the risk of cancer: importance of kinds of tobacco. Lion, IARC, 1984. (Scientific Publication, n. 57).

11. FRANCO, E.L. \& CAMPOS, N. A microcumputer program for multiple regression by conditional and unconditional maximun likelihood methods. Am. J. Epidemiol, 129: 439-44, 1989.

12. GRAHANS, S.; MARSHALL, J.; HAUGHEY, B; BRASURE, J.; FREUDENHEIM, J.; ZIELEZNY, M.; WILKINSON, G.; NOLAN, J. Nutritional epidemiology of cancer of the esophagus. Am.J.Epidemiol, 131: 454-67, 1990.

13. HERBERT, J.R. \& KABAT, G.C. Menthol cigarette smoking and oesophageal cancer. Int. J. Epidemiol., 18: 37-44, 1989. 
14. HOSMER, D.M. \& LEMESHOW, S. Applied logistic regression. New York, John Wiley \& Sons, 1989.

15. HSIEN-WEN, H. Etiology and prevention of oesophageal cancer in China. Oncol. Times, 3: 429-33, 1981 .

16. KEMP, I.W.; CLARKE, K.; KINLEN, L.J. Oesophageal cancer and distilleries in Scotland. Brit. Med. J., 304: 1543-4, 1992.

17. MAHBOUBI E.O. \& ARAMESCH, 8. Epidemiology of esophageal cancer in Iran, with special reference to nutritional and cultural aspects. Prev. Med., 9: 613-21, 1980.

18. METTLIN, C.; GRAHAN, S.; PRIORE, R.; MARSHALL, J.; SWANSON, $M$. Diet and cancer of esophagus. Nutr. Cancer, 2: 143-7, 1979.

19. MUNOZ, N. Precursor lesions of esophageal cancer in high-risk populations in Iran and China. Lancet, 876-9, 1982.

20. POTTERN, L.M.; MORRIS, L.E.; BLOT, W.J.; 2IEGLER, R.G.; FRAUMENI, J.F. Esophageal cancer among black men in Washington, D.C.I. alcohol, tobaccco and other risk factors. J. Natl. Cancer Inst., 67: $777-83,1981$

21. ROSEMBURG, J. Epidemiologic and dietary evidence for a specific nutritional predisposition to esophageal cancer. J. Natl. Cancer Inst., 67: 243-51, 1981.

22. RUBIN, P. Cancer of gastrointestinal tract $I$. Esophageal detection and diagnosis. JAMA, 226: $1544-58,1973$.

23. SAMMON, A.M. A case-control sudy of diet and social factors in cancer of the esophagus in Transkei. Cancer, 69: 860-5, 1992.

24. SANKARANARAYANAN, R; DUFFY, S.W.; PADMAKUMARY, G.; MURALIDHARAN NAIR, $S$;; DAY, N.E. Risk factors for cancer of the oesophagus in Kerala, India. Int. J. Cancer, 49: 485-9, 1991

25. SHOBOKSHI, O.; ALBEDEIWY, A.F.; SAAD, A.A Clinic-patological study of oesophageal lesions in western region of Saudi Arabia: Oncol. Times, 3: 434-4, 1981.

26. SILBER, W. Carcinoma of the oesophagus: aspects of epidemiology and aetiology. Proc. Nutr. Soc., 44: 101-10, 1985.

27. STEFANI, E.; MUÑOZ, N.; ESTEVE, J.; VASSALLO, A.; VICTORA, C.G.; TEUCHAMANN, S. Mate drinking, alcohol, tobacco, diet and esophageal cancer in Uruguay. Cancer Res., 50: 426-31, 1990.

28. TSUGANE, S.; SOUZA, J.M.P.; COSTA JUNIOR, M.L,; MIRRA, A.P; GOTLIEB, S.L.D.; LAURENTI, R.; WATANABE, S. Cancer incidence rates among japanese migrants in the city of São Paulo - Brazil, 1969-78. Cancer Causes Control, 1:189-93, 1990.

29. TUYNS, A.J. \& ESTEVE, J. Pipe, comercial handrolled cigarrete smoking in oesophageal cancer. Int.J. Epidemiol, 12: 110-3, 1983.

30. TUYNS, A.J. Alcohol and cancer. Proc. Nutr. Soc., 49: 145-51, 1990.

31. UNIÃO INTERNACIONAL CONTRA O CÂNCER/FUNDAÇÃO ONCOCENTRO DE SÃO PAULO, Manual de oncologia clínica. 5a. ed. Springer-Verlag, 1991.

32. VICTORA, C.G.; MUÑOZ, R.; DAY, N.E.; BARCELOS, L.B.; PECCIN, D; BRAGA, N.M. Hot beverages and oesophageal cancer in southern Brazil: a case-control study. Int.J. Cancer, 39: 710-6, 1987.

33. WYNDER, E. Dietary habits and cancer epidemiology. Cancer, 43: 1955-61, 1979.

34. ZAMBRAMO, C.C. \& GUERRERO, M.H. Câncer de esôfago. Rev. Gastroent. Peru, 11: 18-31, 1991.

\begin{abstract}
The association between esophageal cancer and smoking and drinking hablts, instruction and nutritional factors was examined by means of the utilization of data of a cese-control study wich was undertaken in the city of $S$. Paulo (Brazil). Eighty five cases were compared with two hundred and ninety two hospital controls, with different diseases, including other kinds of cancer. The crude estimations of the odds ratios were calculated for all the variables. Logistic regression was used in the next steps of the analysis. The drinking habit (odds ratio $=3.68 ; 95 \%$ confidence interval (1.74 - 7.78)), the smoking habit [odds ratio $=4.86 ; 95 \%$ confidence interval $(1.95 \cdot 12.13)$ ] and the frequent eating of hot pepper [odds ratio $=2.48 ; 95 \%$ confidence interval (1.46 - 4.23)] are important risk factors for the disease. The estimate of odds ratio for smoking ordinary clgarette was of $3.43(1.31-8.97)$ and for smoking of com straw hand-rolled cigarette was of 4.18 (1.38-12.66).
\end{abstract}

Esophagical neoplosms, epidemiology. Risk factors. 